\title{
Elipsoit Yüzeyinde Temel Ödev Çözümleri Üzerine Bir İnceleme
}

\author{
İbrahim Öztuğ BİLDİRİĊ ${ }^{*}$ \\ ${ }^{1}$ Selçuk Üniversitesi, Mühendislik Fakültesi, Harita Mühendisliği Bölümü, Konya \\ (bildirici@selcuk.edu.tr)
}

\begin{abstract}
Öz
Elipsoit yüzeyinde eğriliklerin değişken olması nedeniyle temel ödev problemlerinin düzlem ve kürede olduğu gibi her koşulda sonuç veren basit bağıntıları yoktur. Jeodezinin tarihsel gelişiminde problem, uygulamada yeterli olduğundan uzaklıkların 100km den küçük olduğu nokta konumlarına yönelik ele alınmış ve çeşitli çözüm yöntemleri geliştirilmiştir. Bilimsel ve teknolojik gelişmeler sonucu uzak noktalar arasında da geçerli çözümler ortaya çıkmıştır. Bu çalışmada kaynaklarda yer alan çok sayıda yöntemden yaygın kullanımı olan üç yöntem ele alınmış birbirleri ile karşılaştırılmıştır. Elde edilen sonuçlar her tür uzunlukta büyük uzunluklar için geliştirilmiş Vincenty yöntemin tercih edilmesi gerektiğini göstermektedir..
\end{abstract}

Anahtar Kelimeler: Matematiksel jeodezi, elipsoit, temel ödev çözümleri

\section{A Review on Fundamental Calculation Solutions on the Ellipsoid Surface}

\begin{abstract}
Because the curvatures of the ellipsoid surface are variable, there is no simple relation that yields results under all conditions, such as the fact that the Fundamental calculation problems are plane and spherical. In the historical development of geodesy, the problem has been dealt with in terms of locations where the distances are $100 \mathrm{~km}$ small, and various solution methods have been developed. Valid solutions have also emerged among the far-reaching points of scientific and technological development. In this study, three methods which are widely used in many of the sources in the sources have been discussed and compared with each other. The results show that the Vincenty method developed for large lengths of any length should be preferred.
\end{abstract}

Keywords: Mathematical geodesy, ellipsoid, fundamental calculations solutions

\section{GİRIȘ}

Temel ödev çözümleri bir yüzey üzerinde iki koordinat sistemi arasında dönüşümden başka bir şey değildir. Birinci temel ödevde bir noktanın dik koordinatları, bir başka noktaya olan doğrultu açısı ve uzaklık biliniyorken ikinci noktanın dik koordinatları bulunur. Burada dik koordinat kutupsal koordinat dönüşümü söz konusudur. İkinci temel ödevde ise iki noktanın dik koordinatları biliniyorken aradaki kenar ve bu kenarın doğrultu açısı bulunur. Problemi küre yüzeyine taşırsak burada coğrafi koordinat kürsel kutupsal koordinat (azimut, büyük daire yay uzunluğu) dönüşümü söz konusudur. Düzlemde ve kürede analitik geometri ve küresel trigonometri yardımıla problem noktaların birbirlerine göre uzaklıklarından bağımsız olarak çözülür. 
Elipsoit yüzeyinde problemin çözümü basit değildir. Uydu jeodezisi öncesi triyangülasyon yöntemi ile ağların oluşturulduğu dönemde noktaların birini görme zorunluluğu nedeniyle uzaklıklar en çok $100 \mathrm{~km}$ olabiliyordu. Bu dönemde $100 \mathrm{~km}$ altı uzaklıklarda geçerli çözüm yöntemleri ortaya çıkmış ve kullanılmıştır. Uydu tekniklerinin jeodezide kullanımı ile noktaların birbirini görme zorunluluğu ortadan kalkmıș ve aralarındaki uzaklıkları binlerce kilometreye ulaşan noktalar arasında da temel ödev çözümlerine gerek duyulmuş ve bunu çözen yöntemler de ortaya çıkmıştır.

Elipsoit yüzeyinde temel ödev çözümleri 4 grupta toplanabilir (Demirel ve Üstün 2013, Grossman 1976).

1. Jeodezik eğrinin diferansiyel denklemleri ile elde edilen Legendre serilerine dayanan yöntemler: Bu kapsamdaki seriler çabuk yakınsamadığından hesaplama güçlükleri söz konusudur. Serilerde bazı kısaltılmalar yaparak daha hesaplamaya elverişli seriler elde edilmiştir. Bunların en çok bilinenleri Screiber'in birinci temel ödev çözümü ve Gauss Ortalama Enlem Yöntemi’dir. Bu yöntemler büyük uzunluklar için sonuç vermez.

2. Jeodezik eğriyi oluşturan noktalar ve kutbun oluşturduğu elipsoidal üçgen ile küresel üçgen arasındaki ilişkiden yararlanılan yöntemler: $\mathrm{Bu}$ yaklaşım büyük uzunluklar için geliştirilmiş olup, en yaygın kullanılanı Vincenty Yöntemi'dir (Vincenty 1975).

3. Elipsoidin düzleme ya da küreye izdüşümü yapılıp, hesaplama bu yüzeylerde yapılır. Daha sonra elipsoide ters izdüşüm ile çözüm elde edilebilir. $\mathrm{Bu}$ grupta uygulamada en çok kullanılanı GaussKrüger Projeksiyonu'nda indirgeme bağıntıları ile yapılan çözümdür (Demirel ve Üstün 2013, s.180).

4. Bazı çözümlerde jeodezik eğri yerine kiriş, normal kesit yayı, loksodrom ya da uç noktalarındaki azimutları bu noktalardan geçen normal kesit eğrisinin azimutlarına eşit olan eğriler kullanılır.

\section{Araştırmanın Amacı}

$\mathrm{Bu}$ çalışma ile temel ödev çözüm yöntemleri bir biri ile karşılaştırılacak, hangi durumlarda hangi yöntemin uygun olduğu irdelenecektir. Uzak noktalar arasında çözüm sağlayan Vincenty yöntemi yakın noktalar arasında da geçerlidir. Ancak bu yöntemin hesap yükü daha fazladır. Noktalar arası uzaklığa bağlı olarak Vincenty yöntemine altenatif daha az hesap yükü olan yöntemlerin kullanılabilirliği irdelenecektir.

$\mathrm{Bu}$ çalışmada kullanılan notasyon Ek A'da verilmiştir.

\section{TEMEL ÖDEV ÇÖZÜM YÖNTEMLERİ}

Temel ödev çözümlerinde temel olarak iki hesaplama/dönüşüm söz konusudur. Birinci temel ödev probleminde bir noktanın coğrafi koordinatları (elipsoidal enlem ve boylam), ikinci noktaya olan jeodezik eğri uzunluğu ve jeodezik eğrinin birinci noktadaki azimutu biliniyor iken ikinci noktanın coğrafi koordinatlarının ve ikinci noktadaki azimutun bulunması istenir. Küre ve elipsoitte jeodezik eğri meridyenleri sabit açı ile kesmediğinden jeodezik eğrinin azimutu üzerinde bulunan her noktada farklıdır. İkinci temel ödev çözümünde ise iki noktanın coğrafi koordinatları biliniyorken aralarındaki jeodezik eğri uzunluğu ile jeodezik eğrinin uç noktalarındaki azimutların bulunması istenir (Şekil 1). Kısaca özetlemek gerekirse temel ödev çözümleri aşağıdaki gibi ifade edilir.

Birinci Temel Ödev:

Verilenler: $\varphi_{1}, \lambda_{1}, \alpha_{1}, S$ İstenenler: $\varphi_{2}, \lambda_{2}, \alpha_{2}$ İkinci Temel Ödev:

Verilenler: $\varphi_{1}, \lambda_{1}, \varphi_{2}, \lambda_{2}$ İstenenler: $S, \alpha_{1}, \alpha_{2}$

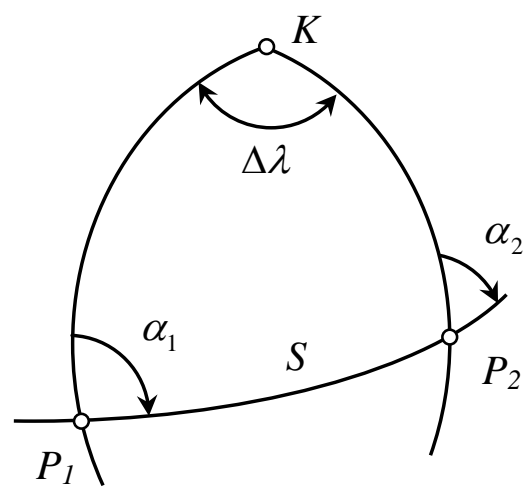




\section{Şekil 1: Temel ödev çözümü}

$\mathrm{Bu}$ başlık altında küçük ve büyük uzaklıklarda kullanılan üç yöntem ele alınacaktır. Tüm bağıntılarda açı birimi radyan olarak alınmıştır. İkinci noktadaki azimut ise $P_{1} P_{2}$ doğrultusunda alınmıştır (Şekil 1).

\section{Schreiber'in Birinci Temel Ödev Çözümü}

Schreiber, Legendre serilerinde kısaltma sağlamak için jeodezik eğri uç noktaları ile ikinci noktadan birinci noktadan geçen meridyene inilen dik ayağı noktasının $\left(P_{f}\right)$ oluşturduğu dik üçgenden yararlanılır. $\mathrm{Bu}$ üçgen bu bölgede elipsoide yakınsayan bir kürede küresel dik üçgen kabul edilir. Büyük uzunluklar için geçerli bir yöntem değildir (Grossman 1976).

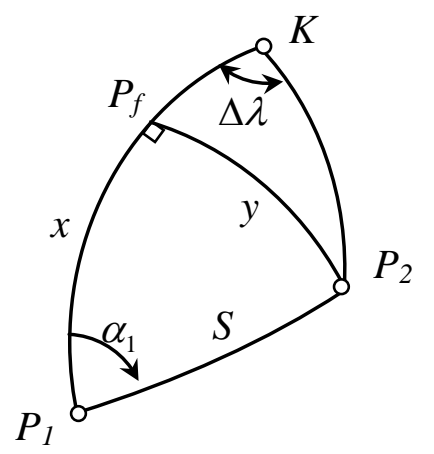

Şekil 2: Schreiber'in birinci temel ödev çözümü

$$
\begin{aligned}
& u=S \cos \alpha_{1} \\
& v=S \sin \alpha_{1} \\
& x=u\left(1+\frac{v^{2}}{3 R_{G 1}^{2}}\right) \\
& y=v\left(1-\frac{u^{2}}{6 R_{G 1}^{2}}\right) \\
& \varphi_{f}=\varphi_{1}+V_{1}^{2}\left(\begin{array}{l}
\frac{x}{N_{1}}-\frac{3 \eta_{1}^{2} t_{1} x^{2}}{2 N_{1}^{2}} \\
-\frac{\eta_{1}^{2} x^{3}\left(1-t_{1}^{2}+\eta_{1}^{2}-5 \eta_{1}^{2} t_{1}^{2}\right)}{2 N_{1}^{3}}+\ldots
\end{array}\right) \\
& \varphi_{2}=\varphi_{f}+V_{f}^{2}\left(\begin{array}{l}
-\frac{t_{f} y^{2}}{2 N_{f}^{2}} \\
+\frac{t_{f} y^{4}}{24 N_{f}^{4}}\left(1+3 t_{f}^{2}+\eta_{f}^{2}-9 \eta_{f}^{2} t_{f}^{2}\right)
\end{array}\right)
\end{aligned}
$$

$$
\lambda_{2}=\lambda_{1}+\frac{1}{\cos \varphi_{f}}\left(\begin{array}{c}
\frac{y}{N_{f}}-\frac{t_{f}^{2} y^{3}}{3 N_{f}^{3}} \\
+\frac{t_{f}^{2} y^{5}\left(1+3 t_{f}^{2}\right)}{15 N_{f}^{5}}
\end{array}\right)
$$

\section{Gauss Ortalama Enlem Yöntemi}

Gauss ortalama enlem yönteminde jeodezik eğrinin ortasında bulunan noktadan yararlanılarak Legendre serilerinde kisaltmalar yapılmıştır. $\mathrm{Bu}$ şekilde elde edilen Gauss ortalama enlem bağıntıları, ortalama enlem, boylam ve azimut,

$$
\varphi=\frac{\varphi_{1}+\varphi_{2}}{2}, \lambda=\frac{\lambda_{1}+\lambda_{2}}{2}, \alpha=\frac{\alpha_{1}+\alpha_{2}}{2}
$$

olmak üzere,

$$
\begin{aligned}
& \Delta \varphi=\frac{u}{M}\left(\begin{array}{l}
1+\frac{2+3 t^{2}+2 \eta^{2}}{24 N^{2}} v^{2}+ \\
\frac{\eta^{2}\left(t^{2}-1\right)}{8 N^{2}} u^{2}
\end{array}\right) \\
& \Delta \lambda=\frac{v}{N \cos \varphi}\left(\begin{array}{c}
1+\frac{t^{2}}{24 N^{2}} v^{2}- \\
\frac{2+7 \eta^{2}-9 \eta^{2} t^{2}}{24 N^{2}} u^{2}
\end{array}\right) \\
& \Delta \alpha=\frac{v t}{N}\left(\begin{array}{c}
1+\frac{2+t^{2}+2 \eta^{2}}{24 N^{2}} v^{2}+ \\
\frac{2+7 \eta^{2}+9 \eta^{2} t^{2}}{24 N^{2}} u^{2}
\end{array}\right)
\end{aligned}
$$

şeklindedir. Birinci temel ödev probleminde ortama değerler (8) bilinmediğinden iteratif olarak çözüm gereklidir. Başlangıç değeri olarak $\varphi=\varphi_{1}, \quad \alpha=\alpha_{1}$ alınıp, (9) ve (11) eşitliklerinden iteratif olarak $\varphi, \alpha, \Delta \varphi$ ve $\Delta \alpha$ belirlenir. Elde edilen son değerler (10) eşitliğinde yerine konularak çözüm gerçekleştirilir.

İkinci temel ödevde ortalama değerler (8) belli olduğu için yöntem daha kullanışlıdır (Demirel ve Üstün 2013, Grossman 1976). Bu amaçla ortalama enlem bağıntılarında $u$ ve $v$ (3) parametreleri çekilerek düzenleme yapılır. 


$$
\begin{aligned}
& u=M \Delta \varphi\left(\begin{array}{l}
1-\frac{2+3 t^{2}+2 \eta^{2}}{24}(\Delta \lambda \cos \varphi)^{2} \\
-\frac{\eta^{2}\left(t^{2}-1\right)}{8 V^{4}} \Delta \varphi^{2}+\ldots
\end{array}\right) \\
& v=N \Delta \lambda \cos \varphi\left(\begin{array}{l}
1-\frac{1}{24}(\Delta \lambda \sin \varphi)^{2} \\
+\frac{1+\eta^{2}-9 \eta^{2} t^{2}}{24 V^{4}} \Delta \varphi^{2}+\ldots
\end{array}\right) \\
& \Delta \alpha=\Delta \lambda \sin \varphi\left(\begin{array}{c}
1-\frac{1+\eta^{2}}{12}(\Delta \lambda \cos \varphi)^{2} \\
+\frac{3+8 \eta^{2}}{24 V^{4}} \Delta \varphi^{2}+\ldots
\end{array}\right)
\end{aligned}
$$

$\tan \alpha=\frac{v}{u}$

$\alpha_{1}=\alpha-\frac{\Delta \alpha}{2}$

$\alpha_{2}=\alpha+\frac{\Delta \alpha}{2}$

$S=\sqrt{u^{2}+v^{2}}$

\section{Vincenty Yöntemi}

Büyük uzunluklarda çözüm sağlayan bu yöntemde yarıçapı ekvator yarıçapı kadar olan bir yardımcı küreden yararlanılır. Jeodezik eğri ile yardımcı kürede oluşan büyük daire yayı arasında ilișki kurularak oluşturulan iç içe eşitliklere dayalı bir çözüm yapılmıştır (Vincenty 1975).

\section{Birinci Temel Ödev}

İlk olarak birinci noktanın enlemi $\left(\beta_{1}\right)$, yardımcı küre üzerinde ekvatordan birinci noktaya kadar açısal büyük daire yayı uzunluğu $\left(\sigma_{1}\right)$ ve jeodezik eğrinin ekvatordaki azimutu $\left(\alpha_{\mathrm{ek}}\right)$ hesaplanır.

$$
\begin{aligned}
& \tan \beta_{1}=\frac{1}{\sqrt{1+e^{\prime 2}}} \tan \varphi_{1} \\
& \tan \sigma_{1}=\frac{\tan \beta_{1}}{\cos \alpha_{1}} \\
& \sin \alpha_{e k}=\cos \beta_{1} \sin \alpha_{1}
\end{aligned}
$$

$A$ ve $B$ büyüklükleri hesaplanır.

$$
\bar{u}^{2}=e^{\prime 2} \cos ^{2} \alpha_{e k}
$$

$$
\begin{aligned}
& A=1+\frac{\bar{u}^{2}}{16384}\left\{4096+\bar{u}^{2}\left[-768+\bar{u}^{2}\left(320-175 \bar{u}^{2}\right)\right]\right\} \\
& B=\frac{\bar{u}^{2}}{1024}\left\{256+\bar{u}^{2}\left[-128+\bar{u}^{2}\left(74-47 \bar{u}^{2}\right)\right]\right\}
\end{aligned}
$$

$\Delta \sigma$ parametresi, $\sigma$ başlangıç değeri,

$$
\sigma=\frac{S}{b A}
$$

alınarak, $\Delta \sigma$ parametresindeki değişim $10^{-14}$ değerinden küçük oluncaya iteratif olarak aşağıdaki bağıntılarla hesaplanır.

$2 \sigma_{m}=2 \sigma_{1}+\sigma$

$\Delta \sigma=B \sin \sigma\left\{\cos 2 \sigma_{m}+\frac{1}{4} B\left[\cos \sigma\left(-1+2 \cos ^{2} 2 \sigma_{m}\right)\right.\right.$

$\left.\left.-\frac{1}{6} B \cos 2 \sigma_{m}\left(-3+4 \sin ^{2} \sigma\right)\left(-3+4 \cos ^{2} 2 \sigma_{m}\right)\right]\right\}$

$\sigma=\frac{S}{b A}+\Delta \sigma$

$\mathrm{Bu}$ şekilde $\sigma_{m}$ ve $\sigma$ parametreleri de iteratif olarak belirlenmiş olur. İkinci noktanın enlemi, $\tan \varphi_{2}=\frac{\sin \beta_{1} \cos \sigma+\cos \beta_{1} \sin \sigma \cos \alpha_{1}}{(1-f)\left[\sin ^{2} \alpha+\left(\sin \beta_{1} \sin \sigma-\cos \beta_{1} \cos \sigma \cos \alpha_{1}\right)^{2}\right]^{\frac{1}{2}}}$

boylam1,

$$
\begin{aligned}
& \tan \Delta \omega=\frac{\sin \sigma \sin \alpha_{1}}{\cos \beta_{1} \cos \sigma-\sin \beta_{1} \sin \sigma \cos \alpha_{1}} \\
& C=\frac{f}{16} \cos ^{2} \alpha_{e k}\left[4+f\left(4-3 \cos ^{2} \alpha_{e k}\right)\right]
\end{aligned}
$$$$
\left.\Delta \lambda=\Delta \omega-(1-C) f \sin \alpha\left\{\begin{array}{l}
\sigma+C \sin \sigma \\
\cos 2 \sigma_{m} \\
+C \cos \sigma\left(-1+2 \cos ^{2} 2 \sigma_{m}\right)
\end{array}\right]\right\}
$$$$
\lambda_{2}=\lambda_{1}+\Delta \lambda
$$

ikinci noktadaki azimut,

$$
\tan \alpha_{2}=\frac{\sin \alpha_{e k}}{-\sin \beta_{1} \sin \sigma+\cos \beta_{1} \cos \sigma \cos \alpha_{1}}
$$

şeklinde hesaplanır.

\section{Ikinci Temel Ödev}

İkinci temel ödev çözümünde ilk olarak ilk olarak iki noktanın indirgenmiş enlem değerleri hesaplanır. 


$$
\begin{aligned}
& \tan \beta_{1}=\frac{1}{\sqrt{1+e^{\prime 2}}} \tan \varphi_{1} \\
& \tan \beta_{2}=\frac{1}{\sqrt{1+e^{\prime 2}}} \tan \varphi_{2}
\end{aligned}
$$

Yardımcı kürede boylam farkı değeri $(\Delta \omega)$, başlangıç değeri,

$\Delta \omega=\Delta \lambda$

alınarak değişim $10^{-14}$ den küçük oluncaya kadar aşağıdaki bağıntılar tekrar edilerek iteratif olarak hesaplanır.

$$
\begin{aligned}
& \sin ^{2} \sigma=\left(\cos \beta_{2} \sin \Delta \omega\right)^{2}+\left(\cos \beta_{1} \sin \beta_{2}-\sin \beta_{1} \cos \beta_{2} \cos \Delta \omega\right)^{2} \\
& \cos \sigma=\sin \beta_{1} \sin \beta_{2}+\cos \beta_{1} \cos \beta_{2} \cos \Delta \omega \\
& \sin \alpha_{e k}=\cos \beta_{1} \cos \beta_{2} \sin \Delta \omega / \sin \sigma \\
& \cos 2 \sigma_{m}=\cos \sigma-2 \sin \beta_{1} \sin \beta_{2} / \cos ^{2} \alpha_{e k} \\
& C=\frac{f}{16} \cos ^{2} \alpha_{e k}\left[4+f\left(4-3 \cos ^{2} \alpha_{e k}\right)\right] \\
& \Delta \omega=\Delta \lambda+(1-C) f \sin \alpha_{e k} \\
& \left\{\sigma+C \sin \sigma\left[\cos 2 \sigma_{m}+C \cos \sigma\left(-1+2 \cos ^{2} 2 \sigma_{m}\right)\right]\right\}
\end{aligned}
$$

Jeodezik eğri uzunluğu, $A, \quad B$ ve $\Delta \sigma$ parametreleri (20) ve (21) bağıntılarıyla hesaplandıktan sonra,

$S=b A(\sigma-\Delta \sigma)$

bağıntısı ile bulunur. Jeodezik eğrinin azimutları aşağıdaki bağıntılardan hesaplanır.

$$
\begin{aligned}
\tan \alpha_{1} & =\frac{\cos \beta_{2} \sin \Delta \omega}{\cos \beta_{1} \sin \beta_{2}-\sin \beta_{1} \cos \beta_{2} \cos \Delta \omega} \\
\tan \alpha_{2} & =\frac{\cos \beta_{1} \sin \Delta \omega}{-\sin \beta_{1} \cos \beta_{2}+\cos \beta_{1} \sin \beta_{2} \cos \Delta \omega}
\end{aligned}
$$

İlgili bağıntılardan görüldüğü üzere Vincenty Yöntemi hesap yükü fazla ve iteratif hesaplamalara duyarlı bir yöntemdir. Büyük ya da küçük tüm jeodezik eğri uzunluklarında geçerlidir.

\section{ARAȘTIRMA}

$\mathrm{Bu}$ başlık altında Schreiber ve Gauss yöntemlerinin hangi koşullar altında yeterli doğrulukta çözüm sağladığı irdelenecektir. Bu amaçla belli uzaklıklarda bir nokta etrafında elipsoit yüzeyinde bir çember oluşturan $1^{\circ}$ azimut farkıyla noktalar oluşturulmuştur.
Elipsoit yüzeyinde eğriliklerin değişimi enlem ve yöne bağl1 olduğundan boylam sabit tutulup yukarıdaki hesaplama $10^{\circ}-80^{\circ}$ enlem aralığında $10^{\circ}$ enlem farkları ile tekrar edilmiştir. Bu şekilde değişik enlemlerde ve yönlerde çok sayıda nokta elde edilmiştir. $\mathrm{Bu}$ noktaların Vincenty yöntemine göre koordinatları doğru kabul edilerek, Schreiber ve Gauss Ortalama Enlem yöntemlerinden elde edilen değerlerle farklarından yararlanılarak standart sapmalar hesaplanmıştır.

Matematiksel jeodezide coğrafi koordinatlarda 0.0001", azimutta 0.001" doğrulukta hesaplama istenir (Demirel ve Üstün 2013, s.104). Schreiber yöntemi ile elde edilen ikinci noktanın coğrafi koordinatları ve azimutunun standart sapma değerleri tablo 1'de görülmektedir. $\mathrm{Bu}$ yöntem ikinci noktadaki azimut $\left(\alpha_{2}\right)$ değerleri açısından $50 \mathrm{~km}$ üzeri kenarlarda yeteri kadar doğru değildir. Coğrafi koordinatlar açısından ise $90 \mathrm{~km}$ uzaklıklara kadar yeterli doğruluk sağlamaktadır.

Gauss Ortalama Enlem Yöntemi için yapılan test sonuçları Tablo 2'de görülmektedir. İkinci noktadaki azimut açısından $100 \mathrm{~km}$ ye kadar kenarlarda yeterli doğruluk sağlanmaktadır. Coğrafi koordinatlar açısından bakıldığında $70 \mathrm{~km}$ kadar kenarlarda elde edilen standart sapmalar uygundur. Tablo değerlerinden görüldüğü üzere iki yöntem doğruluk açısından farklı sonuçlar vermiştir.

Tablo 1: Schreiber Yöntemi Test Sonuçları

\begin{tabular}{ccccc}
\hline$S_{\varphi}^{\prime \prime}$ & $S_{\lambda}^{\prime \prime}$ & $S_{\alpha}^{\prime \prime}$ & $\begin{array}{c}\text { Nokta } \\
\text { Say1s1 }\end{array}$ & $\begin{array}{c}\text { Uzaklık } \\
(\mathrm{m})\end{array}$ \\
\hline 0.000003 & 0.000002 & 0.001280 & 2880 & 50000 \\
0.000009 & 0.000006 & 0.003184 & 2880 & 60000 \\
0.000022 & 0.000018 & 0.006886 & 2880 & 70000 \\
0.000050 & 0.000046 & 0.013443 & 2880 & 80000 \\
0.000101 & 0.000104 & 0.024271 & 2880 & 90000 \\
0.000191 & 0.000218 & 0.041197 & 2880 & 100000 \\
0.000339 & 0.000426 & 0.066525 & 2880 & 110000 \\
0.000574 & 0.000787 & 0.103093 & 2880 & 120000 \\
0.000930 & 0.001384 & 0.154338 & 2880 & 130000 \\
0.001455 & 0.002338 & 0.224368 & 2880 & 140000 \\
0.002208 & 0.003811 & 0.318033 & 2880 & 150000 \\
\hline
\end{tabular}

Kaynaklarda her iki yöntem için de 100km'e kadar uzaklıklarda kullanılabileceği bilgisi yapilan test ile tam olarak doğrulanmamaktadır. $\mathrm{Bu}$ iyimser değerlerin orta enlemli bölgelerde yapılan hesaplamalara dayandığı düşünülebilir. Burada yapılan analiz 
daha geniş bir enlem aralığında gerçekleştirilmiştir.

Tablo 2: Gauss Ortalama Enlem Yöntemi Test Sonuçları

\begin{tabular}{ccccc}
\hline$S_{\varphi}^{\prime \prime}$ & $S_{\lambda}^{\prime \prime}$ & $S_{\alpha}^{\prime \prime}$ & $\begin{array}{c}\text { Nokta } \\
\text { Say1s1 }\end{array}$ & $\begin{array}{c}\text { Uzaklık } \\
(\mathrm{m})\end{array}$ \\
\hline 0.000009 & 0.000030 & 0.000031 & 2880 & 50000 \\
0.000022 & 0.000075 & 0.000078 & 2880 & 60000 \\
0.000048 & 0.000164 & 0.000170 & 2880 & 70000 \\
0.000094 & 0.000320 & 0.000332 & 2880 & 80000 \\
0.000169 & 0.000578 & 0.000600 & 2880 & 90000 \\
0.000286 & 0.000982 & 0.001020 & 2880 & 100000 \\
0.000461 & 0.001588 & 0.001648 & 2880 & 110000 \\
0.000713 & 0.002464 & 0.002557 & 2880 & 120000 \\
0.001066 & 0.003694 & 0.003833 & 2880 & 130000 \\
0.001546 & 0.005377 & 0.005578 & 2880 & 140000 \\
0.002185 & 0.007632 & 0.007917 & 2880 & 150000 \\
\hline & & & &
\end{tabular}

\section{SONUÇ}

$\mathrm{Bu}$ çalışmada elipsoit yüzeyinde geliştirilmiş temel ödev çözüm yöntemlerinden Schreiber, Gauss Ortalama Enlem ve Vincenty yöntemleri ele alınmıştır. Büyük uzunluklar için geliştirilmiş yöntemler küçük uzunluklar için de geçerli sonuçlar verir. $\mathrm{Bu}$ çalışmada Schreiber ve Gauss Ortalama Enlem yöntemleri Vincenty yöntemi ile karşılaştırılmıştır. Her iki yöntemin de genel kanının aksine $100 \mathrm{~km}$ ye kadar yeterli doğrulukta sonuç vermediği, 50-70km uzaklıklara kadar güvenilir oldukları belirlenmiştir. $\mathrm{Bu}$ sonuçlara göre daha fazla hesap yükü getirmesine rağmen Vincenty yönteminin hem uzak hem de yakın uzaklıklarda kullanımının uygun olduğu görülmektedir.

\section{KAYNAKÇA}

Demirel, H. ve Üstün, A. (2013) Matematiksel Jeodezi, Yayınlanmamış Ders Notu, http://atlas.selcuk.edu.tr/1205429/dokuman lar/jeodezi2013.pdf

Grossman, W. (1976). Geodatische Rechnungen and Abbildungen in der Landesvermessungen, 3. Auflage. Konrad Wittwer, Stuttgart, (s 14), 63.

Vincenty, T. (1975). Direct and inverse solutions of geodesics on the ellipsoid with application of nested equations. Survey review, 23(176), 88-93.
Richardus, P., ve Adler, R. K. (1972). Map projections for geodesists, cartographers and geographers, New Holland, Amsterdam. 


\section{EK A: NOTASYON ve KISALTMALAR}

$a, b$

$c=\frac{a^{2}}{b}$

$f=\frac{a-b}{a}$

$e=\sqrt{\frac{a^{2}-b^{2}}{a^{2}}}$

$e^{\prime}=\sqrt{\frac{a^{2}-b^{2}}{b^{2}}}$

$\varphi$

$\lambda$

$S$

$\alpha$

$\beta$

$\Delta \omega$

$\sigma$

$t=\tan \varphi$

$\eta^{2}=e^{\prime 2} \cos ^{2} \varphi$

$V=\sqrt{1+e^{\prime 2} \cos ^{2} \varphi}$

$M=\frac{c}{V^{3}}$

$N=\frac{C}{V}$

$R_{G}=\frac{c}{V^{2}}$
Elipsoit

yarıçapları

Kutup eğrilik

yarıçapı

Basıklık

Birinci dış

merkezlik

İkinci dış

merkezlik

Enlem

(elipsoidal)

Boylam

Jeodezik eğri

uzunluğu

Azimut

İndirgenmiş

enlem

Yardımcı bir küre

üzerinde boylam

fark1

Kürede büyük

daire yay

uzunluğu (açısal)

Kisaltma

Kisaltma

Kisaltma

Meridyen eğrilik

yarıçapı

Çapraz eğrilik

yarıçapı

Gauss küresi

yarıçapı 